\title{
DATA QUALITY CHECK AND VISUAL ANALYSIS OF CORS STATION BASED ON ANUBIS SOFTWARE
}

\author{
Yan Xiao ${ }^{1,2}$, Maohua Yao ${ }^{3, *}$, Shihua Tang ${ }^{1,2}$, Haifeng Liu ${ }^{4}$, Pengwei Xing ${ }^{1,2}$, Yan Zhang ${ }^{1,2}$ \\ ${ }^{1}$ College of Geomatics and Geoinformation, Guilin University of Technology, No.319 Yan'shan Street, Guilin, Guangxi 541006, \\ China - yan@glut.edu.cn \\ ${ }^{2}$ Guangxi Key Laboratory of Spatial Information and Geomatics, Guilin University of Technology, No.12 Jian'gan Road, Guilin, \\ Guangxi 541006, China - (gzhou,yuetao)@glut.edu.cn \\ ${ }^{3}$ Geomatics Center of Guangxi, No.5 Jianzheng Road, Nanning, Guangxi 530023, China - 835956050@qq.com \\ ${ }^{4}$ School of Geomatics, Wuhan University, No 129 Luoyu Road, Wuhan, Hubei 430079, China - 2018102140017@whu.edu.cn
}

Commission I, WG I/8

KEY WORDS: CORS, data quality, TEQC, Anubis, visualization

\begin{abstract}
:
Aiming at the observation data of CORS multi-mode data quality and inspection in CORS station, based on the observation data of a CORS station in Guilin, Guangxi, the paper used TEQC and Anubis software to check the data quality from data utilization, cycle slip ratio and multipath. The results show that the three quality inspection indicators of Anubis software are better than TEQC software, which proves that Anubis' data quality inspection results are effective and reliable. On this basis, using Anubis drawing tools to perform quality inspection and visual analysis from satellite constellation, signal band and observation data itself, it shows that Anubis' visual content is more elaborate than TEQC, and users can more intuitively understand and control the data quality of CORS station.
\end{abstract}

\section{INTRODUCTION}

With the continuous development and application of GNSS technology, users have put forward higher requirements for the real-time and accuracy of navigation and positioning. Continuous operation reference system (CORS) is a dynamic, continuous, rapid and high precision to obtain spatial geographic data and geographical characteristics of the modern spatial information infrastructure. The quality of CORS station data is one of the main factors affecting the accuracy of CORS RTK measurement. Therefore, the CORS station needs to perform quality inspection on the GNSS observation data during the operation and maintenance process. GNSS data quality analysis mainly includes data utilization, cycle slip ratio, multipath effect and signal-to-noise ratio. They can comprehensively reflect the satellite availability, station environment impact, receiver performance and other aspects, and are the premise and guarantee of data post-processing (Yang, Z., 2010; Yu, W. K., 2010).

TEQC (Translate, Edit, Quality Check, Coordinate), which is commonly used in GNSS data processing by UNAVCO Facility developed for geoscientific research service of the public free data processing software, can be used to solve the GNSS data processing problems such as GPS, GLONASS, BDS (Estey, L. H., 1999; Jia, Z. G., 2013). However, the software is poorly interactive and does not have visualization, and there are limitations in visualization with drawing software (such as cumbersome operations, weak compatibility, and low analysis content).To this, this paper introduces a software that can be used for multi-mode data quality inspection and visualization-Anubis, taking GNSS observations of CORS station in Guangxi Guilin as an example, adopted the software of TEQC and Anubis, respectively, to include GPS, GLONASS and BDS navigation satellite system CORS station observation data analysis of quality inspection, and the data quality inspection indicators of the two software were compared to verify the reliability of Anubis. On this basis, Anubis' visualization tools are used to analysis the satellite constellation, signal band and observation data quality indicators of the observation data of the CORS station.

\section{GNSS DATA QUALITY INDICATOR ANALYSIS}

\subsection{Data utilization ratio analysis}

Data utilization rate can not only reflect the availability and integrity of data in the measurement section, but also reflect the stability of receiver signals, which is an important indicator to measure the quality of data. It is necessary to timely analysis the influencing factors of the external environment and the receiver and find out the reasons timely to ensure the normal operation of the observation data. Data utilization ratio is defined as follows:

$$
R=N_{1} / N_{0}
$$

where $\mathrm{R}$ is the data utilization ratio, $N_{1}$ represents the number of observations received, $N_{0}$ represents the number of satellite observations that can be received.

\subsection{Cycle slip ratio analysis}

In the GNSS measurement observation, the Doppler counter's whole-week count is temporarily interrupted, and the part of the carrier phase observation with system deviation and less than one full week remains the correct phenomenon, called the cycle slip (Miao, Y., 2011). The cycle slip ratio is a method of statistics by observing the proportion of observable data in

\footnotetext{
* Corresponding author: Yao Maohua E-mail:835956050@qq.com
} 
weekly epochsz (Tian, Q., 2017). In addition to using the ionospheric residual method for the detection and evaluation of cycle slip, the cycle slip can also be expressed by o/slps or CSR.

$$
\begin{aligned}
& o / \operatorname{slps}=\frac{o}{\operatorname{slps}} \\
& C S R=\frac{1000}{o / \operatorname{slp} s}
\end{aligned}
$$

where $o$ is the number of observations, slps is the number of weeks hops.

\subsection{Data utilization ratio analysis}

The multipath effect is caused by the interference between the reflected satellite signal and the direct satellite signal, which will cause loss of the accuracy of the observation value, and in severe cases will cause the signal to lose lock (Guo, J. Y., 2014a). In the analysis of GNSS data quality indicators, multipath can not only directly reflect the environmental quality around the station, but also is one of the important indicators of GNSS observation data quality.

The multipath effect is divided into pseudorange multipath effect and carrier phase multipath effect. Due to the small value of carrier phase multipath effect, it is customary to use pseudorange multipath effect to reflect the quality of observation data (Guo, J., 2014b). The pseudorange multipath effect can be obtained by combining pseudorange observation and carrier phase observation. The formula for calculating the multipath effect of L1 and L2 carriers is as follows:

$$
M P 1=P_{1}-\left[1+\frac{2}{\alpha-1}\right] \varphi_{1}+\left[\frac{2}{\alpha-1}\right] \varphi_{2}
$$

$$
M P 2=P_{2}-\left[\frac{2 \alpha}{\alpha-1}\right] \varphi_{1}+\left[\frac{2 \alpha}{\alpha-1}-1\right] \varphi_{2}
$$

where $P_{1}$ is the pseudorange observations on the L1 band, $P_{2}$ is the pseudorange observations on the $\mathrm{L} 2$ band, $\varphi_{1}$ is the $\mathrm{L} 1$ carrier phase observation, $\varphi_{2}$ is the L 2 carrier phase observation, $\alpha$ is the L1, L2 two-band frequency and the square of the ratio.

\subsection{Signal to noise ratio analysis}

$\mathrm{SNR}$, defined as the ratio of signal power $\mathrm{S}$ to noise power $\mathrm{N}$, can be used to measure the signal capability of GNSS receiver and reflect its signal tracking and capturing performance. The larger the signal to noise ratio, the better the quality of the observed signal (Xu, K. K., 2016). For general measurement receivers, the signal-to-noise ratio of the outdoor GNSS received signal is approximately in the range of $35-55 \mathrm{dBHz}$. According to its numerical classification, the signal above $40 \mathrm{dBHz}$ is called a strong signal, while the signal below $28 \mathrm{dBHz}$ is called a weak signal.

\section{EXAMPLE ANALYSIS}

\subsection{CORS station data quality check}

The experimental data selected the GNSS observation data of the GLLG station on the 189th day of a CORS station in Guilin, Guangxi. The station receiver is TRIMBLE NETR9, the antenna is TRM59900.00, and the data sampling interval is 30s. Using the 2019 version of TEQC and Anubis software to quality check the GLLG station data, from data utilization ratio,

\begin{tabular}{|c|c|c|c|c|c|c|c|c|c|}
\hline Software & Station & Satellite & Ratio/\% & $\mathrm{o} / \mathrm{slps}$ & $\mathrm{MP} 1 / \mathrm{m}$ & $\mathrm{MP} 2 / \mathrm{m}$ & $\mathrm{MP} 5 / \mathrm{m}$ & $\mathrm{MP} 7 / \mathrm{m}$ & $\mathrm{MP} 8 / \mathrm{m}$ \\
\hline \multirow{4}{*}{ TEQC } & \multirow{4}{*}{ GLLG } & GPS & \multirow{4}{*}{87} & \multirow{4}{*}{969} & 0.31 & 0.38 & 0.42 & - & - \\
\hline & & GLONASS & & & 0.46 & 0.49 & - & - & - \\
\hline & & Galileo & & & 0.75 & - & - & 0.82 & 0.74 \\
\hline & & BDS & & & 0.29 & - & - & 0.26 & - \\
\hline \multirow{4}{*}{ Anubis } & \multirow{4}{*}{ GLLG } & GPS & \multirow{4}{*}{89.82} & \multirow{4}{*}{1141} & 0.28 & 0.31 & 0.35 & - & - \\
\hline & & GLONASS & & & 0.44 & 0.40 & - & - & - \\
\hline & & Galileo & & & 0.18 & - & 0.29 & 0.31 & 0.11 \\
\hline & & BDS & & & - & 0.26 & - & 0.22 & - \\
\hline
\end{tabular}
cycle slip ratio, multipath effect and other aspects to verify the reliability of Anubis. The TEQC and Anubis data quality check results are shown in Table 1 below:

Table 1. TEQC \& Anubis quality inspection results

According to the provisions of the Chinese Crustal Movement Observation Technical Regulations and the data quality inspection analysis of IGS (International GNSS Service), the data utilization rate is not less than $80 \%$. For multipath effects, MP1 is less than $0.5 \mathrm{~m}$ and MP2 is smaller than $0.75 \mathrm{~m}, \mathrm{MP} 5$, MP7, MP8 do not provide the corresponding reference standard, more than half of the CSR value is less than 5, that is, the value of o /slps is greater than 200 (Nie, Z. S., 2013;Chen, C.X., 2014). Based on this standard, it can be seen from Table 1 that the TEQC quality inspection results are compared with the Anubis quality inspection results: from the data utilization index, the evaluation of the utilization rate of the two software are almost the same, and even Anubis data utilization rate is slightly higher, indicating that satellite observation data can be used more effectively. From the cycle slip ratio indicator, the larger the o/slps value, the smaller the cycle slip, and the number of cycle slips of Anubis is greater than that of TEQC, indicating that Anubis cycle slip detection and repair ability is strong. From the multipath effect index, MP1 and MP2 of the two software are basically identical, and both meet the reference standard (since no reference standard is provided for MP5, MP7 and MP8, no comparative analysis is conducted here). In summary, Anubis and TEQC software were compared data quality indicators such as data utilization rate and cycle slip ratio, indicating that Anubis software can more effectively use satellite observation data, indicating that Anubis data quality inspection results are effective and reliable.

\subsection{Anubis visual analysis}

In order to more intuitively analysis the quality of GNSS observation data, in view of Anubis software data quality inspection result is reliable and effective, TEQC software does not have visualization. This paper used Anubis software from satellite number, celestial trajectory, satellite elevation angle, signal channel and other aspects of the GNSS multi-mode data for visual analysis, through its visual graphics can be used to monitor the observation data in all aspects. 

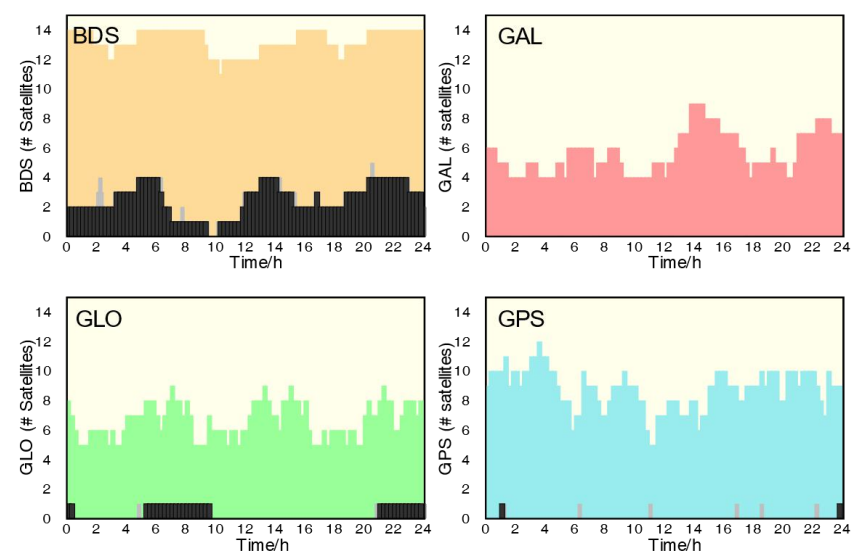

Figure 1. Multi/single satells and bands
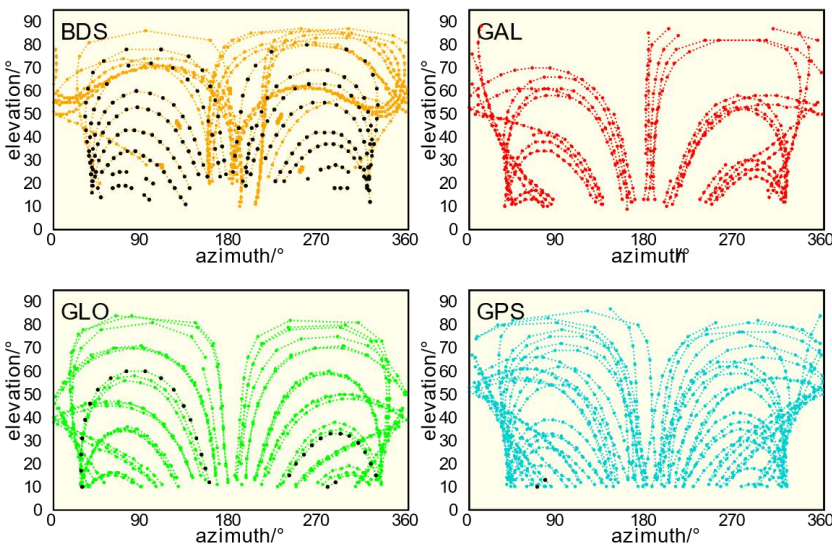

Figure 2. Satellite sky tracks

Figure 1 shows the number of visible satellites at the corresponding moments of each satellite. It can be seen from the figure that the number of visible satellites in BDS and GPS is more than Galileo and GLONASS throughout the day. The number of visible satellites of BDS satellites is large, but it is accompanied by Single frequency satellite. Figure 2 shows the sky trajectory of each satellite. The satellite azimuth is the $\mathrm{X}$ axis and the elevation angle is the $\mathrm{Y}$ axis. The elevation cut-off angle of each satellite is $10^{\circ}$. Due to the early and basic development of GPS and GLONASS construction, the satellite sky trajectory is evenly distributed throughout the world. However, the sky trajectory of BDS is gradually distributed around the world. The Beidou 3 system has not been completed, the single-frequency satellite phenomenon appears in the trajectory. It is precisely because the Galileo satellite developed late, the number of visible satellites is small, resulting in a sparse and uneven distribution of sky trajectories.
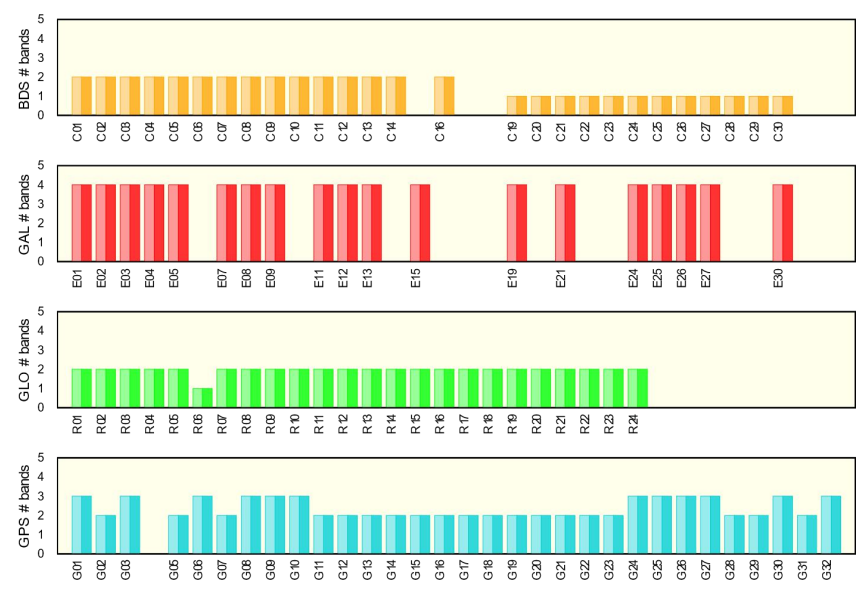

Figure 3. Satellite chart of each constellation
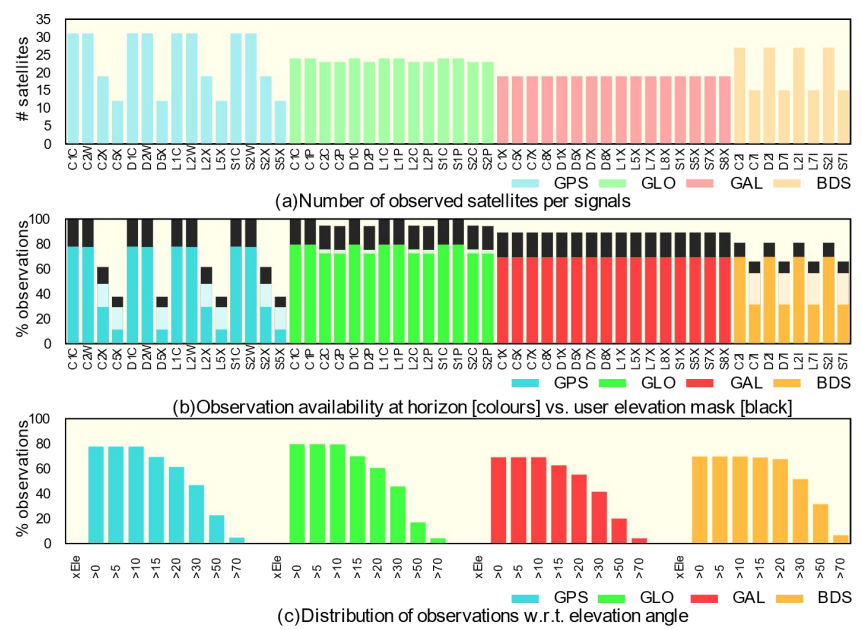

Figure 4. Statistics of the number of observation satellites

Figure 3 shows the satellite statistics of each system. It can be seen from the figure that the total number of satellites of the observed BDS is 27 , and in combination with Figure 1, the number of BDS satellites observed on the GLLG station can reach more than 12 on the same day. The total number of GPS satellites is 31 , and the number of GPS satellites that can be observed on the GLLG station is more than 7 on the same day. It can be seen that the utilization rate of the same satellite in domestic BDS is higher than other systems. Figure 4(a) shows the number of satellites observed in the corresponding band of each system, and Figure 4(b) increases the observed elevation angle of the corresponding band. GPS satellite number or observation Angle, C1 and C2, D1, D2, code and S1, S2, L1 and L2 carrier frequencies observed GPS other wavelengths more than the number of satellites, the BDS C2 and D2, L2 carrier and S2 band number of satellite observed is also more than in any other band BDS, reflecting these band signal stability, utilization rate is higher than the other bands, while the observations of other bands in other systems are comparable. Figure 4(c) shows the number of visible satellites at different elevation angles. 

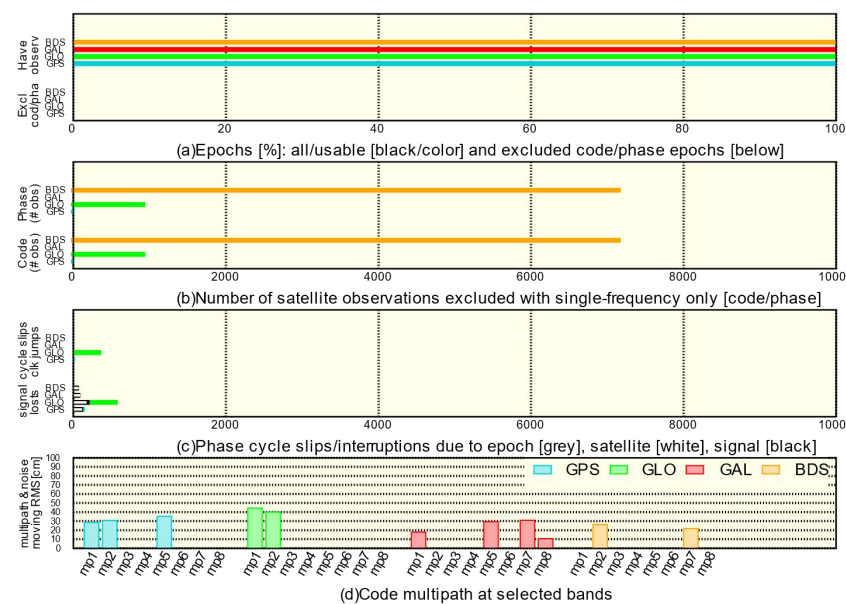

Figure 5. Overall statistical chart of observed data quality

Figure 5 shows the statistical summary information of data quality of each satellite system, including data utilization rate, data rejection ratio, single frequency observations rejected, number of cycle hops caused by epochs, satellite loss of lock and signal disturbance, and multipath effect. As can be seen from Figure 5(a), the full number of observed epochs on the day of the GLLG station reached $100 \%$. To ensure the quality of the observed data, Anubis software eliminated and counted the single-frequency observations. It can be seen from figure $5(\mathrm{~b})$ that Anubis deleted the observation data of about 7600 BDS and 1000 GLONASS. The reason may be that the visible satellite of BDS on that day was a single-frequency satellite, and the visible satellite of GLONASS at some moments was a single-frequency satellite. As can be seen from Figure 5(c), there are fewer signal interruptions in GPS, Galileo, and BDS, which may be due to changes in the observed environment. In addition, the GLONASS satellite observations showed a phenomenon of cycle slip and loss of lock. Finally, Figure (d) shows the RMS value of the selected frequency band. From the figure, it can be found that the RMS values of GPS, Galileo and BDS in the day observation of the GLLG station are equivalent, while the RMS value of GLONASS is slightly larger.

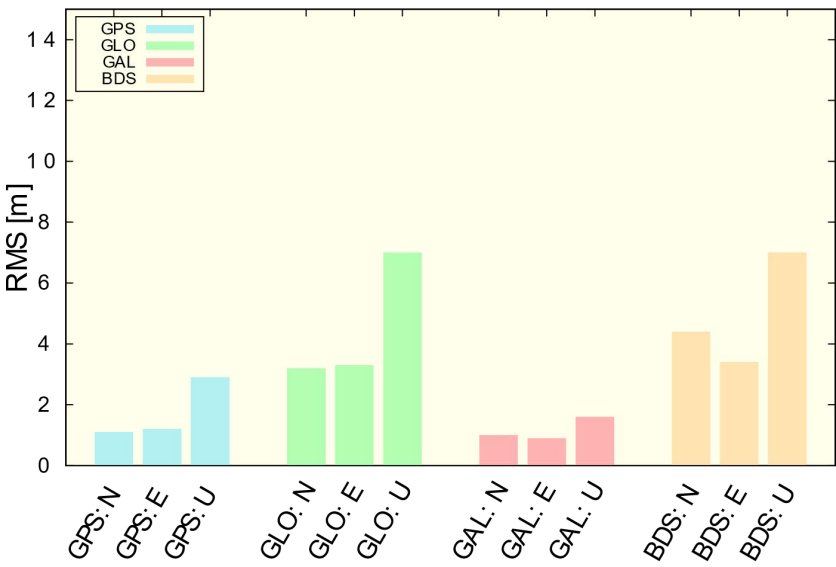

(a) Standard positioning

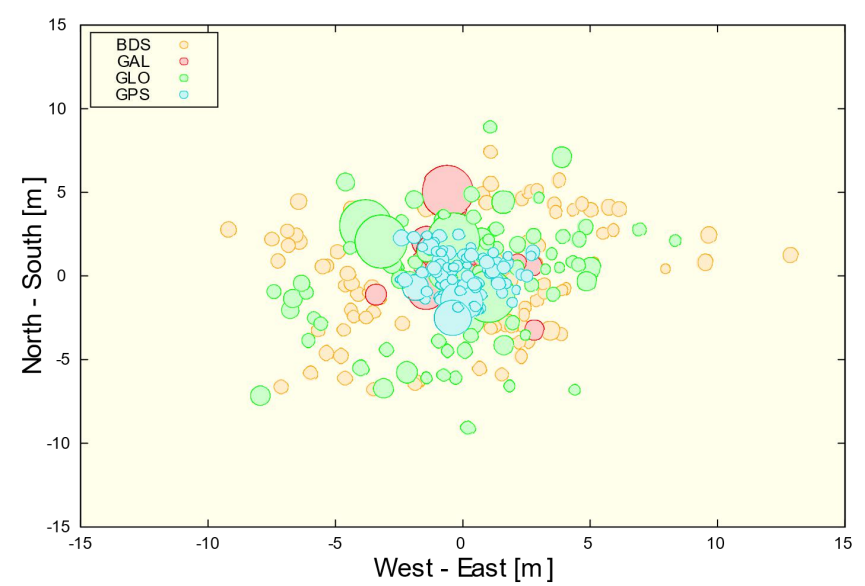

(b) Deviation map of each orientation

Figure 6. Schematic diagram of positioning error

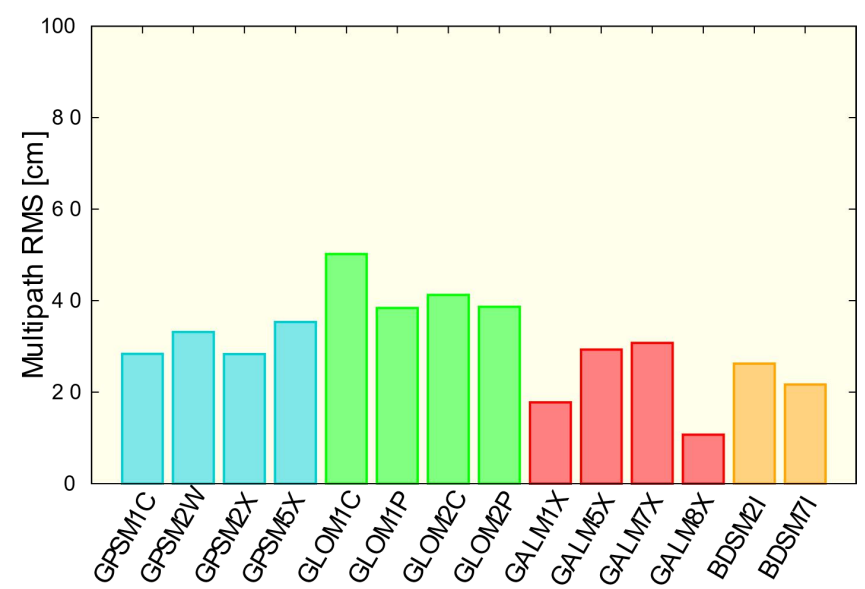

(a)Code multipath

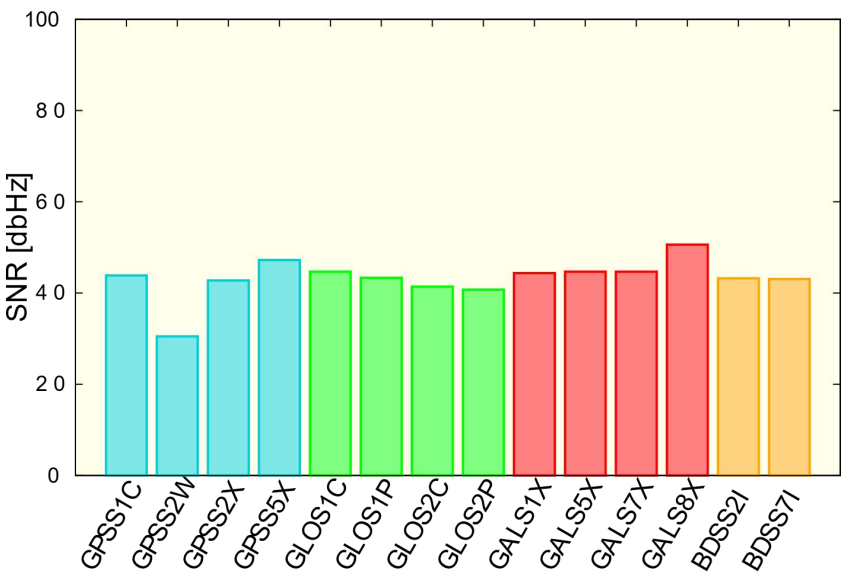

(b) Signal-to-noise ratio

Figure 7. Quality inspection results 


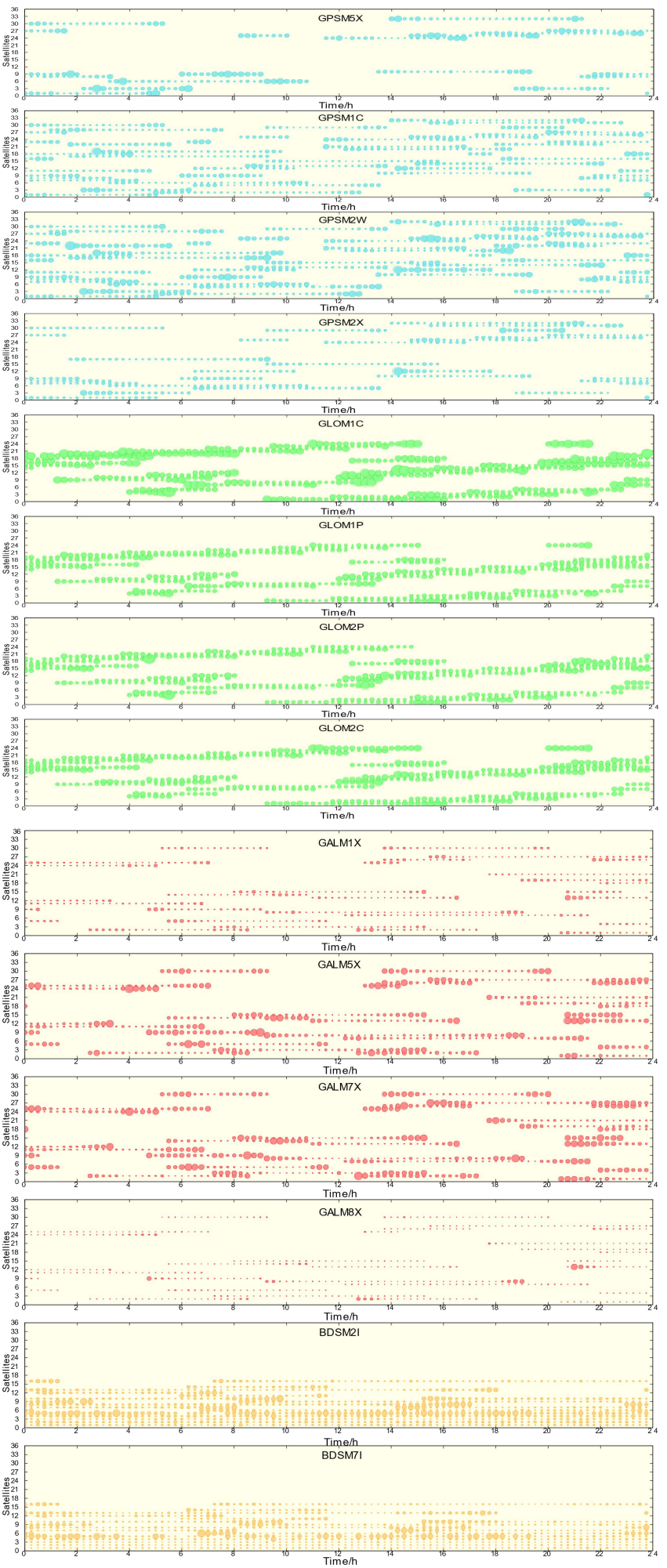

Figure 8. Schematic diagram of multipath effect time series in different bands

Figure 6(a) is a statistical diagram of the positioning accuracy of each position. It can be found from the figure that the positioning accuracy of GPS and Galileo is better. In addition, the positioning errors of each satellite in the $\mathrm{U}$ direction are larger than other directions. Figure 6(b) shows the deviation bubble diagram with a zero deviation center, and the farther the bubble is from the center, the larger the deviation. It can be seen from the figure that the points of GPS and Galileo are relatively small relative to the concentration, indicating that the deviations are relatively small, while the points of GLONASS and BDS are relatively scattered, especially the points of GLONASS are not uniform, indicating that the deviations are large and the performance related instability.

Figure 7(a) is a code multipath statistical graph. In general, the order of the RMS values of each satellite is Galile $<$ BDS $<$ GPS $<$ GLONASS. Figure 7 (b) shows the SNR diagram of each satellite. It can be found from the figure that the SNR of the observed values is basically above $40 \mathrm{dBHz}$, indicating that the signal received by GLLG station is strong and the data quality of the measuring station is relatively stable.

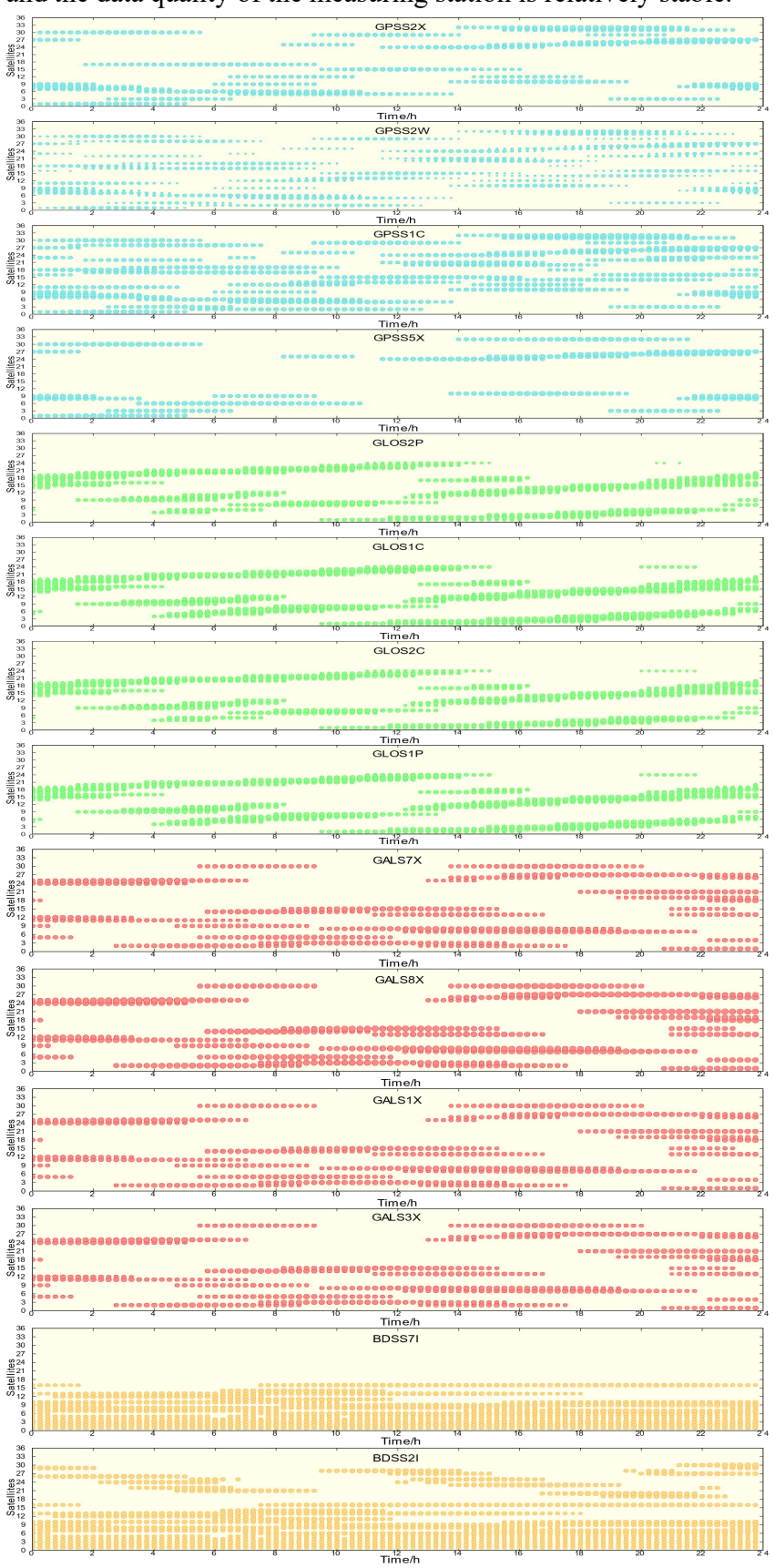

Figure 9. Schematic diagram of signal-to-noise ratio time series in different bands

Figure 8 shows a sequence diagram of multipath variation of different frequency bands of each satellite with time. As the satellite elevation angle rises and falls, the multipath effect of 
each satellite basically shows a trend of large at both ends and small in the middle.

Figure 9 reflects the change of signal-to-noise ratio with time. The denser the points in the figure, the higher the signal-tonoise ratio reflects and the better the data quality of the measuring station. It can be seen from Figure 9 that the signalto-noise ratio of each satellite shows a trend of larger in the middle and smaller at both ends, which is related to the change of height angle during the satellite lifting process. Generally speaking, when the satellite elevation angle rises and falls, the signal-to-noise ratio is relatively small, and as the satellite elevation angle increases, the signal-to-noise ratio gradually increases. The larger the signal-to-noise ratio, the better the corresponding signal quality, the higher the observation accuracy and the better the data quality.

\section{CONCLUSION}

With the continuous development of CORS stations, users' requirements for data accuracy have been constantly improved. In order to ensure the quality of data quality of CORS stations, GNSS observation data should be checked for quality. This paper used TEQC and Anubis software to check the observation data of a CORS station in Guilin, Guangxi, from the data utilization ratio, cycle slip ratio and multipath. By comparing the results of the two software quality checks, it is concluded that Anubis software can more efficient use of satellite observations and Anubis data quality inspection results are effective and reliable. In order to understand the data quality of the CORS station more intuitively, on the basis of this, Anubis is used to visually analysis the data quality inspection results. The results show that compared with TEQC, Anubis software can visually analysis GNSS multi-mode data more conveniently and in detail, and use its visual graphics to monitor the quality of observation data in all directions, such as satellite systems in Guilin. The number of visible satellites is the same (the number of visible satellites in BDS is the highest), and the proportion of data observed in different height angles of the CORS station in Guilin, as well as the utilization rate of observation data and cycle slip. In addition, the multipath and signal-to-noise ratio time series diagram can be used to analysis the quality of the observed data over time, which provides a reference for local measurement operation scheduling.

\section{ACKNOWLEDGEMENTS}

This paper is financially supported by GuangXi Natural Science Foundation under grant numbers 2018GXNSFAA281279, National Natural Science of China under Grant numbers 41864002, and GuangXi Key Laboratory of Spatial Information and Geomatics Program under Grant numbers 16-380-25-13.

\section{REFERENCES}

Yang, Z., Dai, W. J., Yu, W. K.,2010: Quality analysis of gnss data based on teqc in different environments. Journal of Geodesy and Geodynamics 30(5), 135-139.

Yu, W. K., Dai, W. J., Yang, Z.,2010: GNSS data analysis \& pre-processing software design based on TEQC. Journal of Geodesy and Geodynamics 30(05):81-85.

Estey, L. H., Meertens, C.M.,1999: TEQC: The multi-purpose toolkit for GPS/GLONASS data. GPS Solutions 3(1):42-49.
Jia, Z. G., Nie, Z. S., Chen, Z. S., et al, 2013: Analysis and study on gnss data under ehv transmission environment by using teqc. Applied Mechanics and Materials 475-476, 287-290.

Miao, Y., Sun, Z.W., Wu, S. N., 2011: Error analysis and cycleslip detection research on satellite-borne GPS observation. $J$ Aerospace Eng 24(1):95-101.

Tian, Q., Wang, T.,2017: Quality check and analysis on base station data by TEQC. Geotechnical Investigation \& Surveying 45(2):41-44+60.

Guo, J. Y. , Li, G. W. , Kong, Q. L., et al ,2014: Modeling gps multipath effect based on spherical cap harmonic analysis. Transactions of Nonferrous Metals Society of China 24(6), 1874-1879.

Guo J, Li G, Kong Q, et al. 2014: On site pseudorange multipath effect on GPS surveying. Principle and Application Progress in Location-Based Services. Springer, Cham 107-120.

Xu, K. K., Wu, J.C., 2016: Quality evaluation and analysis on gnss data in red river fault profile surveying. Geotechnical Investigation \& Surveying 44(9),49-54.

Nie, Z. S., Huang, Y., Liu, G., et al, 2013: Quality evolution of data from cors in hubei. Journal of Geodesy and Geodynamics 33(6):53-56+61.

Chen, C. X., Chen, G., Wang, Q. P., et al, 2014: Data examination and analysis for fujian gps observation network. Journal of Geodesy and Geodynamics 34(4):17-20. 